\title{
XXI. Amphibol von Cappuccini di Albano.
}

\author{
Von \\ F. Zambonini in Rom. \\ (Hierzu Taf. VI, Fig. 1-9.)
}

Sehr dürftig sind die Notizen, welche wir über den Amphibol der Albaner Gebirge besitzen. Brocchi ${ }^{1}$ ) erwähnt »tremolite in einigen Urgesteinen des Peperins von Albano und dem Giuturna-See: "tremolite " ist auch von L. de'Medici Spada') erwähnt. G. vom Rath ${ }^{3}$ ) bespricht den Amphibol in verschiedenen Stellen seiner Arbeit.

Wir verdanken G. Strüver ${ }^{4}$ ) werthvolle Notizen über die Anwesenheit des Amphibol in den Auswürflingen, welche er untersuchte; auch er gab aber nichts über die Krystallformen dieses Minerals an.

Da ich einige Auswürflinge des Peperins von Cappuccini di Albano mit sehr glänzenden Amphibolkrystallen zur Verfügung gehabt habe, so glaubte ich, dass eine kurze krystallographische Untersuchung darüber nicht ganz nutzlos wäre, um so mehr, als der Amphibol der Albaner Gebirge der einzige unter den vulkanischen italienischen Fundorten des tyrrhenischen Meeres ist, welcher noch nicht krystallographisch studirt wurde.

Viele Forscher haben den Amphibol des Vesuvs untersucht: A. Scacchi 5 ) erwähnt die Formen $\{001\},\{010\},\{100\},\{110\},\{310\},\{130\}$, $\{021\},\{041\},\{111\},\{111\},\{\overline{2} 21\},\{131\},\{151\},\{131\}$. An den Krystallen

1) Catalogo ragionato di una raccolta di rocce, disposto con ordine geografico per servire alla geognosia d'Italia. Milano 1817, 49, 51.

2) Sopra alcune specie minerali non prima osservate nello stato pontificio: in C. Palomba, Raccolta di lettere etc., Roma $1845,1$.

3) Geognostisch-mineralogische Fragmente aus Italien. I. Theil. Zeitschr. d. d. geol. Gesellsch. 1868, 18, 340 .

4) Studi sui minerali del Lazio. Memorie R. Acc. dei Lincei 1876-1877. Auch diese. Zeitschr. 1877, 1, 225.

5) Catalogo dei minerali vesuviani. Rivista di min. e cristall. ital. 1889, 5, 51. Groth, Zeitschrift f. Krystallogr. Xxxrii. 
der Blöcke der Gegend von Bracciano fand Strüver $\left.{ }^{1}\right\}\{100\},\{010\},\{001\}$, $\{110\},\{021\},\{111\} ;$ Artini $\left.^{2}\right)$ an jenen des Sees von Vico $\{010\},\{001\}$, $\{110\},\{021\},\{\overline{1} 11\},\{131\}$; endlich giebt Fantappiè $\left.{ }^{3}\right)$ für die Krystalle der Vulsinischen Gebirge $\{100\},\{010\}:\{001\},\{110\},\{201\},\{021\},\{111\},\{131\}$.

Die von mir beobachteten Auswürflinge bestehen aus grünlichschwarzem Pyroxen, schwarzem Glimmer, Olivin und Ieucit. Bald bilden die genannten Componenten ein inniges Gemenge, bald herrscht der Glimmer vor, so dass es sich um Glimmerblücke handelt. In diesem Falle zeigt der Glimmer sehr grosse Blätter. In den kleinen Höhlungen der Blöcke oder auch auf dem Glimmer sitzend, kommen sehr schüne Krystalle von Amphibol, Leucit, schwarzem Glimmer, Apatitnädelchen und selten Sodalith, Haüyn, Titanit vor.

Die $A$ patitnädelchen sind nach der IIauptaxe sehr verlängert, bald sind sic vollkommen farblos, bald etwas graulich. Sic sind immer an beiden Enden zerbrochen, und ich habe an ihnen nur das Prisma $\{10 \bar{T} 0\}$ erkannt. Die Messung der Brechungsexponenten gab:

$$
\begin{aligned}
& w_{N a}=1,6391 \\
& \varepsilon_{N a}=1,6346
\end{aligned}
$$

für $\mathrm{Na}$-Licht. Es handelt sich also um Fluorapatit.

Die gefundenen Werthe stehen jenen, welche A. Schrauf am Apatit ron Jumilla fand, sehr nahe:

$$
\begin{aligned}
& \omega_{D}=1,63896, \\
& \varepsilon_{D}=1,63448 .
\end{aligned}
$$

Wir werden sehen, dass der Apatit Latiums auch in den Winkeln jenem von Jumilla verwandt ist.

Apatitkryställchen habe ich auch in den Sanden eines Bächleins gefunden, welches die Via Casilina, $6 \mathrm{~km}$ von Rom, durchschnitt. Diese haben genaue Messungen gestattet, und eines von ihnen zeigt auch Endflächen. In der Verticalzone bemerkt man unbeträchtliche Abweichungen von den theoretischen Werthen; die Winkel zwischen zwei benachbarten Prismenflichen waren an zwei Krystallen:

$$
59^{\circ} 58^{\prime}, 6001^{\prime}, 60^{\circ} 3^{\prime}, 59^{\circ} 57^{\prime}, 60002^{\prime}, 59^{\circ} 55^{\prime}, 6001^{\prime} \text {. }
$$

In dieser Zone habe ich die Formen $\{10 \bar{T} 0\}$ und $\{12 \bar{T} 0\}$ bestimmt.

1) Contribuzioni alla mineralogia del vulcani sabatini. Parte I. Sui proietti minerali vulcanici trovati ad est del lago di Bracciano. Memorie R. Accad. dei Lincei 1885, 1 (4a), seduta 1 marzo. Ausz. diese Zeitschr. 12, 197.

2) Contribuzioni alla Mineralogia dei Vulcani Cimini. Memorie della R. Accad. dei Lincei 1889, 6, 93. Ausz. diese Zeitschr. 20, 170.

3) Su i proictti minerali vulcanici trovati nell' altipiano tufaceo occidentale dei Vulsini, da Farnese a S. Quirico e Pitigliano. Memorie R. Accad. dei Lincei 1898, $2\left(5^{2}\right), 347$. 
Der am Ende ausgebildete Krystall, welcher die Combination $\{10 \bar{T} 0\}$ $\{12 \overline{1} 0\}\{10 \bar{T}\}$ darbietet, ist unvollkommen durchsichtig und von hellkastanienbrauner Farbe. Dies ist eine unter den Krystallen der Albaner Gebirge ungewöhnliche Farbe; auch die Grüsse dieses Krystalles ist etwas beträchtlicher. F. Gonnard und Fròre Adelphey) erwähnen ähnlich gefärbte Krystalle in den senclaves « des Chuquet-Genestoux; sie haben sune petite quantité de chlore gefunden; der Krystall der Albaner Gebirge, wie es aus den gemessenen Winkeln hervorgeht, ist fast chlorfrei.

$$
\begin{gathered}
(10 \bar{T} 0):(10 \bar{T} 1)=49047^{\prime} \\
:(01 T 1)
\end{gathered}
$$

Aus dem ersteren Werthe folgt:

$$
c=0,73228
$$

und

$$
(10 \bar{T} 0):(01 \overline{1} 1)=7109^{\prime} 55^{\prime \prime} \text {. }
$$

Der $\Lambda$ patit der Albaner Gebirge ist, infolge dieser Winkel, dem $\Lambda$ patit von Jumilla vollkommen gleich und steht auch dem Apatit von Kirjabinsk sehr nahe. N. v. Kokscharow fand an ihnen:

$$
\begin{aligned}
(0001):(10 \overline{1} 1) & =40013^{\prime} \text { Jumilla } \\
& =40 \quad 13 \frac{1}{2} \text { Kirjabinsk. }
\end{aligned}
$$

Der erwähnte Krystall ist durch das Fehlen der Basis, sowie durch die anormale Entwickelung der Pyramidenflächen merkwürdig.

In den Blöcken, welche vorherrschend aus grossen Glimmerblättern bestehen, kommen manchmal Leucitkrystalle vor, welche durch ihre Schönheit und ihren. Glanz mit jenen des Vesuvs, von G. vom Rath viele Male beschrieben, vergleichbar sind. Sie sitzen auf dem Glimmer, und oft sind sie etwas gerundet, was von einer begonnenen Schmelzung herrührt. Ihre Entwickelung ist ganz unregelmässig; einige sind der Fig. 454 des Hintzeschen Ilandbuches (aus vom Rath copirt) vollkommen gleich. Bei anderen dieser Leucitkrystalle haben Rhombendodekaëder und Leucitoëder fast dieselbe Grüsse; sehr selten ist $\{110\}$ grösser als $\{211\}$.

In einem Stücke eines solchen Glimmerblockes habe ich einen sehr schönen, fast farblosen, etwas in's Meergrünliche fallenden Haüynkrystall gefunden. Er ist ein einfacher Krystall, nach einer trigonalen Axe verlängert, und zeigt die Combination $\{110\}\{100\}\{211\}$.

Von den zwei letzteren Formen sind aber nur einige Flächen anwesend. Die Winkel zwischen den Dodekaëderflïchen sind genau $=60^{\circ}$, und dies ist um so mehr merkwürdig, weil es in demselben Blocke Titanit- und Amphibolkrystalle giebt, welche starke Störungen zeigen. Mit natürlichen Kanten habe ich zweimal den Brechungsexponenten gemessen; das Mittel der zwei übereinstimmenden Bestimmungen ist:

1) Sur l'apatite de certains enclaves granulitiques du Chuquet-Genestoux, Puyde-Dome. Compt. rend. 1895, 126, 1532. Ref. diese Zeitschr. 32, 633. 


$$
n_{\mathrm{Na}}=1,49748 \text {. }
$$

Dieser Werth ist jenem $\left(n_{N a}=1,4961\right)$, welchen Tschihatscheff 1$)$ am Haüyn von Niedermendig fand, sehr nahe.

Ein sehr schöner, fast farbloser, aber deutlich in's Meergrüne spielender Krystall lieferte einen sehr genauen Werth:

$$
n_{N a}=1,50138 \text {. }
$$

Ich habe ferner den Brechungsexponenten eines blassgrünlichen Haüyn aus den Sanden des oben erwähnten Bächleins der Via Casilina bestimmt. Dieser Krystall zeigt das Rhombendodekaëder; er ist nach zwei parallelen Flächen dieser Form tafelförmig. Ich fand:

$$
n_{N a}=1,5038 \text {. }
$$

K. Zimányi ${ }^{2}$ ) hat am Ilaüyn von Latium einen sehr nahestehenden Werth gefunden: $n_{N_{\iota}}=1,5027$.

Aus einem anderen Auswürflinge erhielt ich ein kleines, aber sehr schönes Sodalithkryställchen. Es ist vollkommen farblos, nach einer trigonalen Axe verlängert, und zeigt die Formen $\{110\},\{111\},\{211\}$. Das Rhombendodekaëder ist vorherrschend; die Winkel sind etwas gestürt (z. B. habe ich $59^{\circ} 54^{\prime}$ und $59^{\circ} 57^{\prime}$ statt $60^{\circ}$ gefunden). $\{211\}$ und $\{111\}$ haben sehr kleine Flïchen. Strüver erwähnt am Sodalith von Galloro $\{110\},\{100\}$, \{111\}. Der Brechungsexponent ist:

$$
n_{v a}=1,4832 \text {, }
$$

was mit den Bestimmungen von Feussner ${ }^{1}$ ) am vesuvischen Sodalith vollkommen übereinstimmt. Der Krystall gab eine deutliche Chlorreaction.

Der Titanit, welcher in den Albaner Gebirgen selten ist, kommt in diesen Auswürflingen ziemlich häufig vor; oft ist er aber in eine erdige, braune Substanz umgewandelt.

Einige Krystalle zeigen die Combination $\{001\}\{100\}\{110\}\{111\}\{111\}$, schon von Strüver erwähnt; sie sind der Fig. 9, Taf. II der Arbeit Strüver's vollkommen gleich. Ich habe aber auch Krystalle gefunden, welche flächenreicher sind; ausser der eben besprochenen Combination habe ich auch die drei folgenden beobachtet:
b) $\{001\}\{100\}\{110\}\{111\}\{111\}\{112\}$,
c) $\{001\}\{100\}\{010\}\{110\}\{111\}\{111\}\{T 12\}$,
d) $\{001\}\{100\}\{010\}\{110\}\{111\}\{111\}\{112\}\{101\}$.

Die drei Formen $\{010\},\{T 01\}$ und $\{112\}$ waren bis jetzt am Albaner Titanit noch nicht gefunden worden; sie sind am Titanit des Sees von Vico, von Artini untersucht, bekannt. $\{010\}$ ist immer sehr klein und selten, ebenso wie $\{(101\}$. Grösser ist $\{112\}$, welches manchmal über $\{$ T11 $\}$ vorherrscht. Diese Krystalle unterscheiden sich von jenen durch Strüver

1) In Rosenbusch, Mikroskopische Physiographie etc. 1885, 268.

2) Die Hauptbrechungsexponenten der wichtigeren gesteinsbildenden Mineralien bei $\mathrm{Na}$-Licht. Diese Zeitschr. 189', 22, 329. 
beschriebenen durch die grössere Entwickelung von $\{100\}$, sowie auch durch die Kleinheit von $\{001\}$.

Aus den Messungen, welche Strüver veröffentlicht hat, scheint es, dass der Albaner Titanit in guter Uebereinstimmung mit den Des Cloizeaux'schen Constanten ist. Er gab:

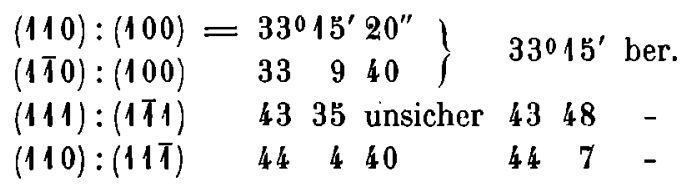

Nach der Arbeit Strüver's hat Arzruni 1) seine Studien über den Amphibol und den Titanit von Ponza und Procida bekannt gemacht. Nach Arzruni sind die Constanten jener Titanite

$$
a: b: c=0,7513: 1: 0,8566 ; \beta=60^{0} 19 \frac{1^{\prime}}{} \text {. }
$$

Dem sublimirten Titanit kommt daher ein etwas anderes Axenverhältniss $\mathrm{zu}$, als den in den älteren massigen Gesteinen und krystallinischen Schiefern vorkommenden Krystallen. Letztere befolgen die von A. Des Gloizeaux und P. v. Jeremejew berechneten Constanten. Da einige meiner Krystalle sehr genaue Messungen gestatten, so habe ich versucht, ob der Titanit der Albaner Gebirge die Constanten von Arzruni oder jene von Des Cloizeaux oder Jeremejew befolgt.

Ich habe die Beobachtung Strü ver's bestätigt, dass nämlich die Winkel $(100):(110)$ und $(100):(1 \overline{1} 0)$ gewöhnlich verschieden sind. Drei schöne Krystalle lieferten:

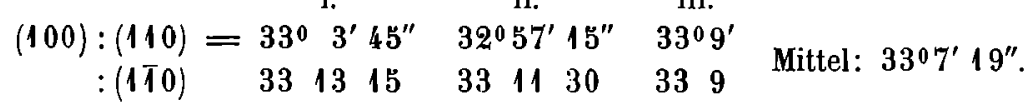

$\Lambda \mathrm{n}$ allen drei Krystallen war (100): $(010)=90^{\circ}$ genau.

Ein anderer Krystall gab:

$$
\left.\begin{array}{rrr}
(001) & :(111)=38^{0} 23^{\prime} \\
:(11 T) & 109 & 28 \\
(111):(11 T) & 71 & 5 \\
(T 1 T):(11 T) & 71 & 11
\end{array}\right\} \text { Mittel: } 71^{\circ} 8^{\prime} .
$$

An einem anderen Krystalle fand ich:

$$
\begin{aligned}
& (100):(T 11)=119^{0} 26^{\prime} \\
& (111):(111) \quad 8440
\end{aligned}
$$

Endlich zwei Kryställchen gaben:

$$
\begin{aligned}
(100):(001) & =60010^{\prime} 25^{\prime \prime} & \text { I. } & \text { Mittel: } 60^{\circ} 11^{\prime} 42^{\prime \prime} . \\
& =6013 & \text { II. } & \\
(001):(\bar{T} 01) & =6611 & \text { II. } &
\end{aligned}
$$

1) Krystallographische Untersuchungen an sublimirtem Titanit und Amphibol. Sitzungsber. d. kgl. Akad. d. Wiss. zu Berlin 1882. Ausz. diese Zeitschr. 8, 296. 
Aus diesen Messungen geht hervor, dass der Albaner Titanit in besserem Einklange mit den Constanten von $\Lambda$ rzruni als mit jenen von Des Gloizeaux ist. Die berechneten Werthe nach beiden Axenverhältnissen sind:

\begin{tabular}{|c|c|c|c|c|}
\hline \multicolumn{2}{|r|}{ Des Cloizeaux: } & \multirow{2}{*}{$\begin{array}{c}\text { Untersch. zwisch. } \\
\text { Mess. u. Rechn.: } \\
y^{\prime} 11^{\prime \prime}\end{array}$} & Arzruni1): & \multirow{2}{*}{$\begin{array}{l}\text { Untersch. zwisch } \\
\text { Mess. u. Rechn.: } \\
0^{\prime} 41^{\prime \prime}\end{array}$} \\
\hline$(100):(110)$ & $=33^{0} 14 \frac{1}{2}^{\prime}$ & & $33^{0} \quad 8^{\prime}$ & \\
\hline : $(001)$ & 6017 & 518 & $60 \quad 19 \frac{1}{2}$ & 748 \\
\hline$(001):(T 01)$ & 6557 & 140 & 6616 & 50 \\
\hline$:(111)$ & $38 \quad 16$ & 7 & $\begin{array}{ll}38 & 23\end{array}$ & - \\
\hline$:(11 T)$ & 10937 & 9 & $109 \quad 22$ & 60 \\
\hline$(111):(11 T)$ & 7121 & 13 & $70 \quad 59$ & 9 \\
\hline$(100):(T 11)$ & 1197 & 190 & 11925 & 10 \\
\hline$(\bar{T} 11):(111)$ & $3 \frac{1}{2}$ & $\begin{array}{c}3630 \\
\Sigma=110^{\prime} 59^{\prime \prime}\end{array}$ & $84 \quad 26 \frac{1}{2}$ & $\begin{array}{c}1330 \\
\Sigma=42^{\prime} 59^{\prime \prime}\end{array}$ \\
\hline & & $\varepsilon=1352$ & & $\varepsilon=522$ \\
\hline
\end{tabular}

Der Amphibol kommt in sehr schönen, aber fast immer kleinen Krystallen vor, welche sehr genaue Messungen geliefert haben. Sie sind vollkommen schwarz, sehr glänzend, nach $e$ verlängert; nicht selten sind sie nach $c$ und $b$ gleich entwickelt. Selten sind Krystalle nach $c$ verlängert und nach. $\{010\}$ tafelförmig. Es giebt auch nadelförmige Krystalle.

Die beobachteten Formen sind folgende:

$\begin{array}{lccl}\{100\} & \infty P \infty & h^{1} & a \\ \{010\} & \infty R \infty & g^{1} & b \\ \{001\} & 0 P & p & c \\ \{110\} & \infty P & m & m \\ \{130\} & \infty R 3 & g^{2} & e \\ \{021\} & 2 R \infty & e^{\frac{1}{2}} & \approx \\ \{041\} & 4 R \infty & e^{\frac{1}{1}} & s \\ \{111\} & -P & d^{\frac{1}{2}} & k \\ \{111\} & P & b^{\frac{1}{2}} & r \\ \{\overline{2} 21\} & 2 P & b^{\frac{1}{4}} & o \\ \{131\} & 3 R 3 & b^{\frac{1}{2}} d^{\frac{1}{4}} g^{1} & i \\ \{151\} & 5 R 5 & b^{\frac{1}{4}} d^{\frac{1}{0}} g^{1} & \varrho\end{array}$

$\{100\}$ ist sehr häufig, aber fast immer sehr klein; sehr selten sind Krystalle, an welchen es gross ist, und nur einer war nach dieser Form tafelförmig. $\{010\}$ fehlt nie, es ist manchmal klein, öfters ziemlich gross und häufig grösser als $\{100\}$. Nicht selten sind Krystalle, welche diese Form ziemlich gross zeigen; selten sind aber jene nach $\{0 \mid 0\}$ tafelförmig. Die Basis ist

1) Aus dem Axenverhältnisse von Arzruni folgen die in der obigen Tabelle gegebenen Werthe; Arzruni berechnete für einige von ihnen etwas verschiedene Werthe. 
gewöhnlich eine der grössten unter den Endflächen; manchmal ist sie aber sehr klein. Das Prisma $\{110\}$ ist an fast allen Krystallen die vorherrschende Form der Zone [001], selten ist es klein. $\{130\}$ ist immer klein und ziemlich häufig.

\{021\} ist eine fast constante Form und hat sehr wechselnde Grösse. $\{041\}$ ist sehr selten; ich fand sie an einem einzigen Krystalle mit einer ziemlich grossen Fläche, welche eine nicht ganz genaue Messung gestattete:

$$
\left.(010):(041)=40^{\circ} 55^{\prime} \text { gem. (ber. } 41020 \frac{3^{\prime}}{4}\right) \text {. }
$$

Diese Form, obwohl seit langer Zeit bekannt, ist am Amphibol sehr selten; H. B. Patton ${ }^{1}$ ) erwähnte sie in neuerer Zeit an den Krystallen des Schriesheimer Diorites, A. Scacchi an jenen des Vesuvs.

Ziemlich häufig ist $\{111\}$, welche aber immer kleine Flächen bietet. (T11) kommt an allen Krystallen vor; ihre Entwickelung ist ganz wechselnd; bald ist sie die grösste, bald die kleinste der Endflächen. $\{\overline{2} 21\}$ ist nicht selten und zeigt gewöhnlich ziemlich grosse Flächen. Auch diese Form ist am Amphibol selten; A. Franzenau ${ }^{2}$ ) beobachtete sie an den Krystallen des Aranyer Berges, Cathrein ${ }^{3}$ ) an jenen von Roda, L. Gentilit) an den Krystallen des Monte Vulture, welche eine merkwürdige Formenähnlichkeit mit jenen von Albano zeigen.

Eine sehr häufige Form ist $\{\overline{1} 31\}$; ich habe sie an fast allen Krystallen bestimmt. Manchmal hat sie sehr kleine Flächen, aber gewöhnlich ist sie entwickelter. Nur einmal wurde $\{\bar{T} 51\}$ beobachtet und genau an demselben Krystalle, welcher $\{041\}$ zeigte. Sie ist eine am Amphibol seltene Form; Franzenau erwähnt sie an den Krystallen des Aranyer Berges.

Was die Combinationen betriff, so muss man bemerken, dass sie wenige sind. Die bemerkenswerthesten sind in den Figg. 1-9, Taf. VI dargestellt. Die häufigste ist $\{100\}\{010\}\{001\}\{110\}\{021\}\{111\}$, gewöhnlich auch mit $\{131\}$; häufig ist auch die Combination $\{100\}\{010\}$ $\{001\}\{110\}\{021\}\{111\}\{111\}$, fast immer mit $\{131\}$. Merkwürdig ist der Krystall Fig. 7, an welchem $\{021\}$ fehlt.

Die Krystalle bilden oft Gruppen in paralleler Stellung; häufig sind die Flächen der Zone [001] tief gestreift parallel der Axe $c$; es giebt aber auch viele Krystalle, an welchen die Streifung fehlt. Alle Endflächen sind sehr eben und glänzend und lieferten ganz genaue Messungen.

1) Hornblende, Oligoklas und Tilanit aus Drusenräumen im Schriesheimer Diorit. N. Jahrb. f. Min. u. s. w. 1887, 1, 261. Ref, diese Zeitschr. 14, 499.

2) Krystallographische und optische Untersuchungen am Amphibol des Aranyer Berges. Diese Zeitschr. 1884, 8, 568.

3) Ueber die Hornblende von Roda. Diese Zeitschr. 1888, 13, 9.

4) Sur l'existence de la hornblende dans les tufs volcaniques du Monte Vulture. Bull. soc. franç. de minér. 1894, 17, 81. Ausz. diese Zeitschr. 26, 220. 
Eine wahre Seltenheit sind symmetrisch ausgebildete Krystalle; meistens, wie aus den Figuren hervorgeht, haben die Flächen derselben Form eine ganz verschiedene Grösse. Nur sehr selten sind die zwei Flächen von $\{021\}$ oder von $\{111\}$ gleich entwickelt; $\{131\}$ hat gewöhnlich eine einzige Fläche. Dies ist auch der Fall an den Krystallen des Trachyts von Montesanto, welche durch P. Francol) beschrieben wurden.

Die Untersuchungen von Arzruni und Franco haben gezeigt, dass der Amphibol des Vesuvs und von Ponza bedeutende Störungen zeigt. Die Krystalle der Albaner Gebirge sind viel regelmässiger. Ziemlich oft ist der Winkel (001): $(010)$ von $90^{\circ}$ verschieden; aber die Differenz ist auf wenige Minuten beschränkt. Die Werthe, an fünf Krystallen gefunden, sind:

$$
90^{\circ} 10^{\prime}, 90^{\circ} 6^{\prime}, 90^{0} 0 \frac{1}{2}^{\prime}, 89^{\circ} 58^{\prime}, 9006 \frac{1}{2}^{\prime} \text {. }
$$

An einem anderen Krystalle maass ich $(001):(010)=89^{\circ} 36 \frac{1}{2}^{\prime} ;$ die $\Lambda$ bweichung von dem berechneten Werthe ist zu gross, und ich habe daher den Krystall sorgfältig gemessen, um festzustellen, ob er asymmetrisch war. Die zahlreichen ausgeführten Messungen haben mich versichert, dass der Krystall geometrisch als monosymmetrisch zu belrachten ist, und dass die constatirten Unregelmässigkeiten auf die auch an anderen vulkanischen Mineralien noch unerklärten Stürungen zurückzuführen sind.

Auch die anderen Krystalle zeigen Abweichungen von der monoklinen Symmetrie, bald ziemlich bedeutend, bald unerheblich.

An zwei Krystallen habe ich gefunden:

$$
\begin{aligned}
& \text { I. }(010):(110)=61^{0} 581^{\prime} \quad(010):(110)=62^{\circ} 7^{\prime} \\
& \text { II. } \quad=65 \text { b } \quad=621
\end{aligned}
$$

An einem dritten:

$$
\text { III. }(001):(021)=29^{0} 38^{\prime} \quad(001):(0 \overline{2} 1)=29033 \frac{1}{2}^{\prime}
$$

An anderen Krystallen:

u. s. w.

$$
\begin{aligned}
& \text { IV. }(040):(\overline{1} 31)=49^{0} 39^{\prime} \quad(0 \bar{T} 0):(\bar{\pi} 31)=49^{0} 30 \frac{1}{2}^{\prime} \\
& \text { V. }:(T 11)=74 \quad 5 \quad:(\text { TT } 1)=7359 \frac{1}{2} \\
& \text { VI. }=74 \quad 10 \quad=7359
\end{aligned}
$$

Man kann nicht zwcifeln, dass die Krystalle vollkommen monoklin sind, denn während an einem Krystalle eine Zone oder eine Winkelgruppe asymmetrisch scheint, sind die anderen Zonen vollkommen monosymmetrisch; ferner sind die constatirten Störungen nicht constant; ich habe z. B. viele Krystalle gefunden, an welchen die Winkel der zwei Flächen (021) und (0יㄴ) zu (001) genau gleich sind; das Gleiche gilt auch für die anderen Formen.

1) Ueber Amphíbol und Sodalith aus dem Trachyt von Montesanto. Diese Zcitschrift 1896, 25, 328. 
Aus den Winkeln

$$
\begin{aligned}
& (001):(110)=76^{\circ} 25^{\prime} 30^{\prime \prime} \\
& (010):(110) \quad 62240 \\
& (001):(021) \quad 293620
\end{aligned}
$$

folgt das Axenverhältniss ${ }^{1}$ )

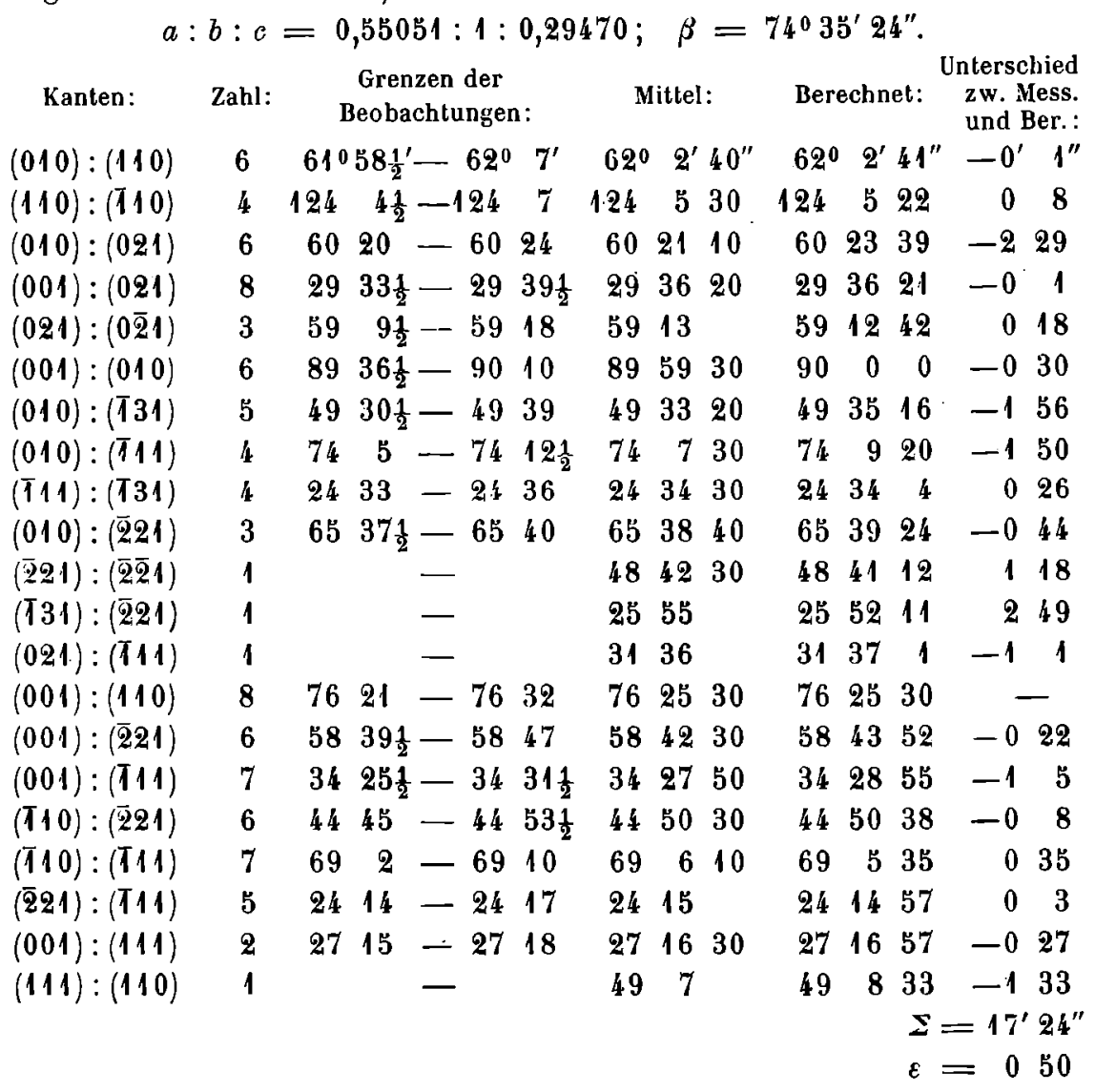

Das Axenverhältniss des schwarzen Amphibols von Cappuccini di Albano besitzt einen gewissen Werth, weil es nicht nur von den Nordenskiöldschen, sondern auch von den anderen bis jetzt berechneten Constanten erheblich abweicht, wie aus folgender Zusammenstellung hervorgeht:

F. Zambonini, Albano $a: b: c=0,55051: 1: 0,29470 ; \beta=74^{\circ} 35^{\prime} 24^{\prime \prime}$ A. Arzruni, Ponza $\quad 0,54556: 1: 0,29353 ; \beta=744830$ A. Franzenau ${ }^{2}$, Aranyer Berg $\quad 0,54812: 1: 0,29455 ; \beta=74397$

\footnotetext{
1) Genauer: $a: b: c=0,550506: 1: 0,2946983 ; \beta=74035 ' 24^{\prime \prime}$.

2) Krystallographische und optische Untersuchungen am Amphibol des Aranyer Berges. Diese Zeitschr. 1884, 8, 568 .
} 
G. Flink ${ }^{1}$, Nordmarken $a: b ; c=0,54152: 1: 0,28857 ; \beta=74048^{\prime} 30^{\prime \prime}$

P. Franco, Montesanto $0,5476: 1: 0,2928 ; \beta=7446$

A. $0 \operatorname{sann} 2)$, Granatilla $\quad 0,5422: 1: 0,2931 ; \beta=7524$

Nils Nordenskiöld $0,54826: 1: 0,29377 ; \beta=75 \quad 2$

Diese Tabelle zeigt, dass der Amphibol der Albaner Gebirge in seinen Winkeln jenem des Aranyer Berges sehr nahe steht. Seine Constanten bieten den grüssten Werth für $a$ und $c$, den kleinsten für $\beta$.

1) Mineralogiska Notiser. Il. Bihang till k. Svenska Vetensk.-Akad. Handl. 1887, 13, II, Nr. 7, 1. Ausz. diese Zeitschr. 15, 90.

2) Beiträge zur Kenntniss der Eruptivgesteine des Cabo de Gata. II. Zeitschr. d. d. geol. Gesellsch. 1891, 43, 697. Ref, diese Zeitschr, 23, 292. 